\title{
The Strength Analysis of the Diagonal Stay of Aircraft Landing Gear Based on ANSYS
}

\author{
Ying XING ${ }^{1, a}$ \\ ${ }^{1}$ Faculty of Mechanical Engineering and Automation, Liaoning University of Technology, Jinzhou \\ China \\ agliuhuaxin@sina.com
}

Keywords: Simulation, ANSYS, Aircraft landing gear, Diagonal stay, Stress

\begin{abstract}
As the landing gear structure is complicated, it is difficult to draw accurate stress and strain distribution through the theoretical calculation. This paper carries on modeling and force analysis to the buffer mechanism of aircraft landing gear, transforms the stress problem dynamic system into static stress problem according to D'Alembert principle and analyzes the diagonal stay of landing gear, and at the same time, analyzes the strength of an aircraft main landing gear by using computer simulation technology and finite element analysis technology, which provides effective basis for damage prediction and practical maintenance.
\end{abstract}

\section{Introduction}

The diagonal stay of landing gear is the essential component of strut-type main landing gear structure [1]. Its role is to control the partial stress of damper strut, restrain the rotation of strut's outer cylinder relative to fuselage and provide torque. The performance, that is good or bad, has a direct impact on the takeoff and landing of aircraft. For landing gear's strength analysis, Yu-zhen CHEN [2] et al. have studied axle's static strength simulation analysis of aircraft landing gear, Xue-hong HE [3] et al. have carried on finite element strength analysis to the four-frame of aircraft landing gear, Xiao-feng WANG [4] et al. have performed static structure optimization to the stay of aircraft landing gear, but researches on dynamic stress analysis of aircraft landing gear are still scanty. This paper researches the dynamic performance simulation of landing gear, carries on the structural strength analysis to the diagonal stay of landing gear using dynamic-static method based on simulation result, which is a critical step in structure strength design and in-depth maintenance work.

\section{Modeling of Landing Gear's Damping Mechanism and Force Result Analysis}

The mathematical model of landing gear is relatively complex. Firstly, in order to make the model simulate actual situation better, fit in with the movement characteristics of landing gear structure and easy to calculate, the origin of coordinate is set on the center of two masses under stop condition. The assumptions made before modeling are as follows:

1) Aircraft's fuselage is a rigid body

2) All the forces of aircraft landing gear are limited in a vertical plane

3) Ignore the offset distance relative to damper's center line

4) The center of elastic support mass is located on the intersection of landing gear strut's center line and trunnion's center line of aircraft's fuselage

5) Inelastic support mass is concentrated on axle ideally

Elastic support mass is the air spring support mass that includes aircraft's fuselage, wing, damper's outer cylinder, etc. Inelastic support mass includes plunger, wheel, brake gear, etc. Based on the assumptions above, a two-mass-spring system is established shown in Fig.1.

Among them, m1: elastic support mass, m2: inelastic support mass, y1: the displacement of elastic support mass, y2: the displacement of inelastic support mass, Fa: the air elasticity of damper, Fh: oleo damping force, Fu: the vertical reaction force of tires. 
The buffer system's kinetic model of aircraft landing gear can be obtained from the physical model and each component force above, and simulation diagram is established based on Matlab Simulink, the simulation result is shown in Fig.2.

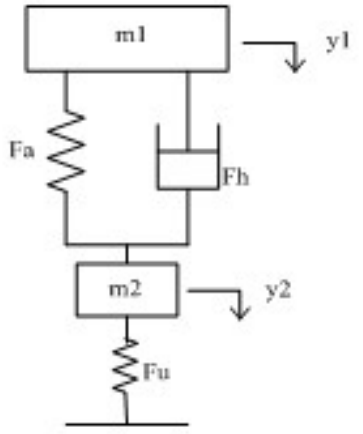

Fig.1 The two-mass-spring system

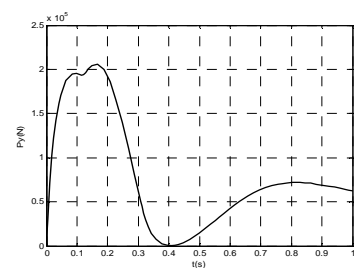

Fig.2 The curve of tires’ vertical force

The figure shows that tires' vertical force changes periodically and dynamically, and the value decreases gradually. Tires' vertical force reaches the maximum at $0.18 \mathrm{~s}$, and the maximum is $2.06 \times 10^{5} \mathrm{~N}$. The diagonal stay's maximum force situation can be obtained from the maximum of tires' vertical force, and it is used as the initial value of diagonal stay's strength finite element analysis.

\section{Force Analysis of Diagonal Stay}

The main landing gear is subjected to the function of concentrated force coming from tires' touchdown point. The force transfers as follow: ground loads are transferred to cylinder rod through tires' touchdown point and two action spots on wheel's center line, cylinder rod balances the ground loads' component force in the direction of cylinder axis through the oil pressure of high-pressure oil-air mixture between cylinder rod and outer cylinder, the ground loads' component force perpendicular to the direction of cylinder axis is transferred to outer cylinder through the interface of cylinder rod and outer cylinder, and torque is transferred to outer cylinder through torque arm. Finally, the load is transferred from outer cylinder to fuselage through diagonal stay and the joint of top connection and fuselage.

Aircraft's landing is a process of dynamic impact. As aircraft's tires touch the ground, the force of landing gear keeps changing continuously. To get when the deformation of diagonal stay is the biggest and where the deformation appears, this paper adopts the dynamic-static method of fluid kinetics and takes the time which impact is maximum as the analytical basis.

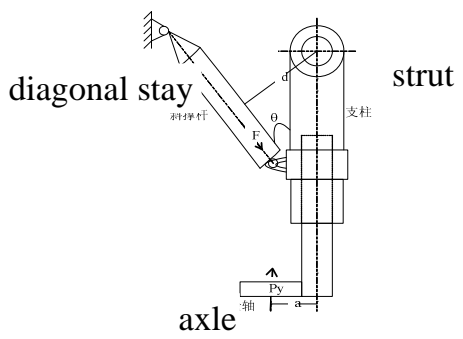

Fig.3 The partial diagram of strut-type landing gear

The relative position between diagonal stay and strut is shown in Fig.3, the vertical distance from the diagonal stay to strut's top hinge is $d$, the angle between diagonal stay and strut is $\theta$, the supporting strength diagonal stay provides to strut is $F$, the distance from axle's center line to strut's center line is a, the vertical force of axle is $P_{y}$, and it is the vertical reaction force $F_{u}$ of tire.

The force of diagonal stay getting from the torque balance equation of strut's top hinge is 


$$
F=\frac{P_{y} a}{d}
$$

And the bending moment of diagonal stay's lower end is $M=P_{y} a$. This paper undertakes an analysis just on the situation of vertical landing, and doesn't analyze taxiing process after landing. Therefore, the bending moment and torque of diagonal stay and strut, which are caused by ground friction force in flying direction, are simplified. When analyzing the force and torque of diagonal stay, only the force state in vertical direction is took into account.

\section{Finite Element Strength Analysis}

Establish Finite Element Model of Diagonal Stay, Load and Analyze. In order to decrease the computational scale and improve accuracy, the model can be pointedly simplified according to the emphasis of analysis when modeling [8]. For instance, the attachments such as lower lock sensor, pipes, etc. are removed which have less impact on the stress of diagonal stay. And to achieve ideal effects when mesh dividing, the influence of welding stress in welds and welding seam is ignored. This way, the result is conservative, and it is of benefit to analysis. The diagonal stay, which is a key component linking up fuselage with landing gears, is shown in Fig.4.

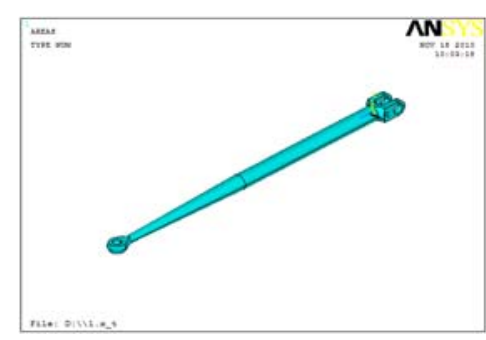

Fig.4 The structural model of diagonal stay

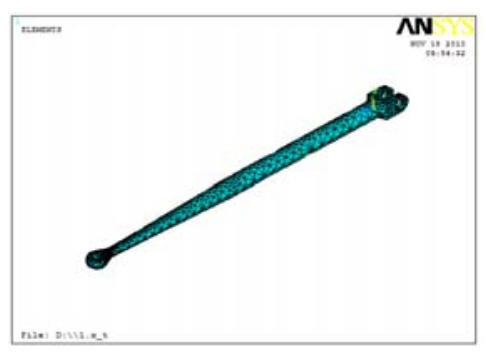

Fig.5 The meshed model of diagonal stay

Select Element Type and Define Material Property. Due to the structure features of diagonal stay, this paper selects eight-node element Solid92. It is tetrahedron element used to analyze 3D solid, which has relatively higher precision. The material of diagonal stay is 40CrNi2Si2MoVA, density is $7.82 \times 10^{3} \mathrm{Kg} / \mathrm{m}^{3}$, elastic modulus is $2.1 \times 10^{11} \mathrm{MPa}$, Poisson's ratio is 0.3 .

Meshing. Owing to the relatively complex structure, the diagonal stay is meshed in free mesh mode, and local meshes are refined according to the actual situation. For instance, larger mesh density is used on the ends of diagonal stay as the force is greater. The use of different mesh density not only assures calculating accuracy, but also reduces the amount of mesh and computation. Finally, 14576 nodes and 8798 elements are generated. The model of diagonal stay after meshing is shown in Fig.5.

Exert Constraint and Load. This paper carries on static analysis to the diagonal stay of landing gear using dynamic-static method, and the number of constraint condition is reduced substantially. The diagonal stay is mainly used to not only reduce the bending moment of strut but also retract and extend landing gear. Therefore, displacement constraints in X direction are exerted on two terminal surfaces of the joint of diagonal stay and fuselage, which limits the revolution of diagonal stay around $\mathrm{Y}$ axis or $\mathrm{Z}$ axis and displacement in $\mathrm{X}$ direction. And displacement constraints in $\mathrm{X}$ direction are exerted on two terminal surfaces of the joint of diagonal stay and landing gear, which limits the revolution of diagonal stay around $\mathrm{Y}$ axis or $\mathrm{Z}$ axis and displacement in $\mathrm{X}$ direction.

As aircraft's landing is a dynamic process and the analysis at one time is static, dynamic-static method of fluid kinetics is adopted. D'Alembert principle of particle system is as follow: whenever particles motion, if mass force is added to each particle, the system is in static state of equilibrium under the action of virtual mass force, realistic active force and ideal constraint force. Within the range of linear elasticity, the stress and strain submit Hooke law. According D'Alembert principle, mass force is added to diagonal stay to make a static analysis on the basis of force analysis. That is, 
the stress and strain of diagonal stay is analyzed at the time when impact is maximum during aircraft's landing.

From the analysis made above, diagonal stay's force reaches the maximum at $0.18 \mathrm{~s}$, and the maximum is $1.03 \times 10^{5} \mathrm{~N}$, tires' vertical force is $2.06 \times 10^{5} \mathrm{~N}$. At this moment, aircraft's landing acceleration is $6.83 \mathrm{~m} / \mathrm{s}^{2}$. Due to the use of dynamic-static method, the load is mass force and loading direction is in the direction opposite of actual acceleration. Material density is needed when mass force is loaded, which has been defined above.

Results Analysis. Solving and the result is shown in Fig.6.

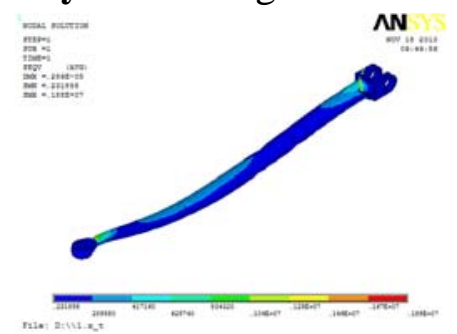

Fig.6 The stress nephogram of diagonal stay

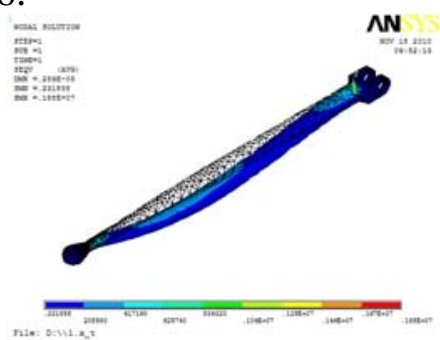

Fig.7 The deformation comparison diagram

From the figure we can see the maximum strength is $0.149 E+7 P a$. From stress nephogram we can see the stress concentration at diagonal stay's lower end is severe and it is easy to be fractured. This illustrates that the rocker is safe in strength, but fatigue failure is still easy to occur on the parts of stress concentration after using for a long time. And the parts of stress concentration should be improved in aircraft overhaul and rocker maintenance, for instance, thicken the thickness of diagonal stay's lower end appropriately. Besides, stress concentration also occurs on upper part of diagonal stay, and it should be checked carefully when maintaining. As seen in Fig.7, at this point, diagonal stay's force is too great. Although diagonal stay doesn't break, bend deformation has occurred. During aircraft landing, the stress of diagonal stay would attenuate periodically. From the analysis above we can see that, after the aircraft landed, diagonal stay must be replaced to ensure the safety of aircraft landing gear.

\section{Conclusions}

This paper mainly achieves the finite element analysis of the diagonal stay of main landing gear. On the basis that the model of aircraft's buffer system is established and simulation result is obtained, the stress problem dynamic system is transformed into static stress problem according to D'Alembert principle. And the force situation of diagonal stay is analyzed according to moment balance principle, the conclusion that stress concentration occurs on the joint of diagonal stay and damper, as well as the joint of diagonal stay and fuselage, is drawn by using the super finite element analysis software, which provides theoretical basis for damage prediction and practical maintenance.

\section{References}

[1] Science and Technology Commission of Min of Aerospace Industry: Strength Design Guide for Aircraft Landing Gears (University of Electronic Science and Technology of China Press, Chengdu 1989)

[2] Yuzhen CHEN and Weijian YU: Machinery. Vol. 33 (2006), p. 33

[3] Xuehong HE, Yingjie ZHANG, liyang XIE, Li HUI. FEM Numerical Analysis on Aircraft Undercarriage Four-frame Structure. in: Chinese Mechanical engineering society, eds. Proceedings of 14th Fatigue \& Fracture Conference. Beijing: Aircraft Strength Research Institute of China, 2008. P. 569

[4] Xiaofeng WANG and Yongjun WANG: Science Technology and Engineering. Vol. 8 (2008), p. 3244 
[5] James N.Daniels. A method for landing gear modeling and simulation with experimental validation. NASA Langley Research Center. Hampton, Virginia.

[6] Youjin SHI and Zhengchang ZHANG: Journal of Southeast University (Natural Science Edition). Vol. 35 (2005), p. 549

[7] Lyle K H, Jackson K E, Fasanella E L. Simulation of aircraft landing gears with a nonlinear dynamic finite element code[R]. AIAA-2000-4049, 2000.

[8] Yong YE, Yanhua HAO and Changhan ZHANG: Mechanical Engineering \& Automation. Vol. 6 (2004), p. 63 